\title{
LA ÉTICA DEL CUIDADO Y SU APLICACIÓN EN LA PROFESIÓN ENFERMERA
}

\section{Loreto García Moyano}

Resumen: Este trabajo tiene como objetivo analizar la utilidad de la ética del cuidado en la profesión enfermera. Para ello será necesario definir los conceptos de "cuidado" y "ética del cuidado" y analizar la posible aplicación práctica de la ética del cuidado en el ámbito de la enfermería española. Se utilizó el método analítico-sintético; revisión bibliográfica, lectura comprensiva, reflexión personal, definición de conceptos y método comparativo.

Palabras clave: ética del cuidado, bioética, enfermería

\section{The ethics of caring and its application in nursing profession}

Abstract: This article has as aim to analyze the usefulness of the ethics of caring in nursing profession. For this it will be necessary to define the concepts of "caring" and "ethics of caring" and analyze the possible practical application of ethics of caring in the nursing field of Spain. The analytic-synthetic method, bibliography review, comprehensive reading, personal reflection, definition of concepts and comparative method were used.

Key words: ethics of caring, bioethics, nursing

\section{A ética do cuidado e sua aplicaçáo na profissáo de enfermagem}

Resumo: Este trabalho tem como objetivo analisar a utilidade da ética do cuidado na profissão de enfermagem. Para isso será necessário definir os conceitos de "cuidado" e "ética do cuidado" e analisar a possível aplicaçáo prática da ética do cuidado no âmbito da enfermagem espanhola. Utilizou-se método analítico-sintético; revisão bibliográfica, leitura compreensiva, reflexão pessoal, definição de conceitos e método comparativo.

Palavras-chave: ética do cuidado, bioética, enfermagem

\footnotetext{
${ }^{1}$ Grupo de Investigación en Bioética, Instituto Aragonés de Ciencias de la Salud, Huesca, España Correspondencia: loretongarcia@hotmail.com
} 


\section{Introducción}

Si pretendiéramos determinar el significado de la profesión enfermera con una sola palabra, esta sería; "cuidado"(1). Pero esta labor no debería ser entendida simplemente como la acción o el arte de proporcionar los instrumentos que sirvan para mejorar o aliviar la enfermedad, sino que, en una visión más amplia, debería entenderse como la acción de proporcionar el mayor bienestar, ya sea físico, psíquico o psicosocial a la persona(2).

Es evidente que la profesión enfermera ha incluido la ética en su plan de estudios. Además, estos profesionales tienen ya presencia en la mayoría de asociaciones científicas de bioética, también participan en los comités éticos y a esto hay que sumar la adopción del Código de Ética de Enfermería por parte de algunos colegios profesionales, así como del llamado Código Deontológico de la Enfermería Española(2-4). Sin embargo, la prioridad de los gestores de nuestro sistema de salud sigue siendo atender a los múltiples avances tecnológicos de los últimos tiempos, así como el reconocimiento legal e institucional de los derechos de los pacientes, la implementación de las políticas de calidad y, en general, la transformación de los sistemas generales de seguridad social. En consecuencia, la mayoría de las reclamaciones de los pacientes se centra en la falta de proximidad y confianza con los profesionales sanitarios.

Parece ser que esta falta de cercanía ha podido surgir, por un lado, por la pérdida justificada de las actitudes paternalistas por parte de los profesionales, así como por la renovada legislación en cuanto a derechos del paciente se refiere, y por último al afán de las últimas décadas por parte de los gestores, investigadores, etc., de aprovechar al máximo los recursos — sobre todo los "materiales" y "cuantificables" — de manera equitativa, buscando una "justicia social” que, en la práctica, contrariamente, pierde la consideración individual y humana de la persona(5).

En síntesis, podríamos decir que la tan buscada excelencia clínica ha mejorado la calidad técnica de los cuidados en cuanto al uso de recursos y prestaciones, e incluso ha ayudado a la mejora en la organización de los cuidados, evaluados por medio de indicadores de obligado cumplimiento por parte de los profesionales. Pero todo esto, a su vez, ha dejado de lado otros aspectos tanto o más importantes, como son el acompañamiento, la empatía, la confidencialidad y, en definitiva, los aspectos que podrían definir una buena relación terapéutica.

Precisamente, si logramos dotar a la profesión de una utilidad no solo técnica sino humana, alcanzaremos, además de la excelencia en el cuidado profesional, la satisfacción de realización personal y colectiva.

Ahora bien, para lograr este objetivo debemos dotar a los profesionales no solo de medios técnicos, sino de herramientas útiles para hacer frente a los problemas o dilemas éticos que surgen en su vida diaria(6). Debemos centrarnos en reconocer los conflictos de valores o problemas éticos con los que nos encontramos, los mecanismos para resolverlos, dejando a un lado nuestros propios intereses. Así, podríamos decir que, aun con la demostrada carga ética de nuestra labor, la enfermería en general no ha sabido tomar un camino definitorio. Además, a esto tenemos que sumar las grandes presiones de las instituciones en cuanto a tareas delegadas y técnicas. La profesión parece tener claro que las decisiones no pueden basarse en unos ideales, en un conjunto de reglas preestablecidas o de los tan famosos principios clásicos éticos(7). Se debe reconocer la importancia de la ética de principios, que ha sido capaz de adentrarse en el ámbito clínico, poniendo encima de la mesa las cuestiones o dilemas éticos por medio de un sistema empírico. No obstante, este modelo no ha sabido, aun con las insistentes demandas por parte de los usuarios, tener en cuenta la humanización de los cuidados. Parece que añadir relaciones humanas a este modelo carece de rigor científico(6).

La ética del cuidado podría convertirse en la respuesta buscada por nuestra profesión, ya que responde a la pregunta fundamental: ¿cuál debería ser la naturaleza de la relación con la persona enferma, y cuáles son las responsabilidades dentro de esta relación?(8). 


\section{Concepto de cuidado}

La palabra "cuidado" viene del latín cogitatus, que significa reflexión, pensamiento, interés reflexivo que uno pone en algo(9). Por tanto podemos decir que el cuidado, el cuidar, parte del interés de alguien por otro, pero no solo de manera afectiva, sino reflexiva y racional.

La actividad de cuidar es toda acción humana que contribuye a la ayuda y solicitud ante la necesidad del otro(1). El cuidado profesional, como labor propia, debería acabar con la dualidad entre las dos conocidas dimensiones del cuidar: la técnica y la humana. Dicha dualidad no se corresponde con la realidad en que vivimos; sería absurdo e imposible omitir o encargarnos tan solo de una de sus dimensiones. Resulta entonces impracticable medir el cuidado en sentido estricto; el cuidar de manera profesional comprende tanto la parte instrumental como la trascendental; no es una sin la otra(10). Esta última parte, la transcendental -o espiritual, si se quiere llamar así-, es difícil de cuantificar, e incluso se podría considerar el intento de hacerlo un absurdo y un fracaso profesional, porque se trata de una aptitud, de una vocación y, en definitiva, de la expresión de una actitud inherente al ser humano.

Resumiendo, el acto de cuidar incluye las tareas más técnicas, es decir, las que garantizan las necesidades más básicas de las personas, y las actividades o actitudes que establecen las relaciones terapéuticas, las cuales permiten la adecuación de las perspectivas de los pacientes con la realidad, así como la propia realización del profesional y sobre todo del paciente(11). Esta relación terapéutica, además de otras actividades no registrables o no cuantificables - como por ejemplo la preocupación, la educación sanitaria, la motivación, la escucha, etc.- , forman parte de los llamados cuidados invisibles o subvalorados(12).

Es entonces al proporcionar dichos cuidados invisibles cuando podemos afirmar que existe la necesidad de un cuidado que va más allá de la necesidad física. Encontramos una necesidad transcendental y espiritual que requiere una respuesta de por lo menos la misma magnitud. El cuidado además es "el resultado de un proceso decisorio de lo que se pretende hacer, de lo que se es capaz de hacer y de lo que se puede llegar a hacer"(13). En consecuencia, el cuidado, como decisión o actitud, tiene un significado y es entonces cuando se hace necesario poseer unos valores que reporten una actitud para llevarlas a cabo(1). Por todo esto, al ejercer de manera oficial, existe un compromiso profesional y ético que diferencia a la actividad enfermera de la actividad espontánea de cuidado(7).

Recapitulando, se podría decir que, al igual que no hay un concepto definido de cuidar, tampoco hay una manera única de hacerlo. Al igual que con las personas, cada relación y cada contexto situacional es también único.

\section{Aproximación a la ética del cuidado}

En 1978, Lawrence Kohlberg, psicólogo, llevó a cabo una investigación sobre el desarrollo moral para comprobar si, al igual que en el desarrollo cognitivo (Jean Piaget), existía un desarrollo moral secuencial y por etapas, que diera como resultado una cierta maduración moral. Aunque este modelo parece ser el más aceptado, sobre todo en cuanto a la certeza de la hipótesis, también la metodología utilizada ha sido objeto de numerosas críticas(14). Quizás la crítica más reveladora es la existencia de un sesgo de género, ya que el psicólogo centró su estudio en los razonamientos de participantes varones $(6,15,16)$. Esto fue lo que impulsó a Carol Gilligan, una de sus discípulas, a investigar más a fondo sobre la cuestión. Hasta entonces no existían investigaciones relacionadas con el desarrollo moral que incluyeran a las mujeres y esto, según Gilligan, era una omisión de verdades y una limitación en el desarrollo del concepto de "condición humana". El objetivo de la investigadora no era otro que "ensanchar la comprensión del desarrollo humano, aprovechando el grupo que se omitió en la construcción de la teoría, para llamar la atención hacia lo que falta en su versión" y, además, proporcionar "una base sobre la cual generar una nueva teoría que, potencialmente, pueda darnos una visión más general de las vidas de los sexos" $(16,17)$.

En sus investigaciones Gilligan afirma que simplemente observando la forma que tienen las personas para hablar de sus vidas y el lenguaje que emplean, pueden deducirse sus conexiones con 
el mundo y cómo actúan en él. De esta manera, mediante la observación, la psicóloga distinguió dos voces o, lo que es lo mismo, dos maneras diferenciadas de hacer frente a las cuestiones morales(17). La psicóloga, relacionando todas estas observaciones, se da cuenta de que existen diferencias entre el pensamiento moral femenino y el masculino $(1,16,18,19)$. No obstante, vale la pena recalcar que en lo que se centra la autora no es en las características biológicas o sexuales, sino en las diferencias del juicio moral en sí.

Parece ser que, en las cuestiones morales relevantes, los hombres se basan en la tradición y la razón, siendo decisiones al fin y al cabo más impersonales; las decisiones de las mujeres parecen estar más relacionadas con los afectos y las relaciones(20). En especial, las mujeres parecen demostrar mucha más sensibilidad hacia los demás y no creen en reglas inamovibles; no juzgan tanto, sino que ven en los conflictos en general un asunto de responsabilidad, dando importancia a las consecuencias de dichos juicios o sus propias acciones, y su moral se basa en el cuidado del otro. Por otro lado, los hombres en general suelen hacer juicios basados en reglas o normas universales, con juicios más claros y menos trascendentales e individualizados, y su moral se basa en los derechos(1). De ahí que la autora relacionara la ética de la justicia con los hombres, y la que llamó ética de la responsabilidad o del cuidado con las mujeres.

Pero ambas visiones de una misma realidad podrían ser perfectamente aceptadas, las resoluciones o puntos de vista más impersonales de los hombres quedarían justificados por una ética de derechos o justicia. En el caso de las mujeres, su percepción ampliada, en la que tienen cabida las relaciones sociales, estaría respaldada por la ética del cuidado y atención. Podría considerarse que los dos puntos de vista buscan una manera justa de resolver los problemas: la ética de los derechos lo hace basándose en la igualdad e imparcialidad, mientras que la ética del cuidado se basa en la igualdad, pero a la luz de una responsabilidad sobre los otros, que descubre y tiene en cuenta la singularidad no solo del que sufre el problema, sino de los demás implicados(1). Entonces, sería honesto afirmar que, aun con sus buenas intenciones, la ética de los derechos o de la justicia abandona $-\mathrm{O}$ al menos deja en segundo plano- dimensiones de la ética, elementos substanciales de la realidad, como las relaciones humanas, los sentimientos, la voluntad, la afectividad, etc. Estos componentes forman parte de un todo, de una realidad que no podemos "fragmentar" para tomar decisiones que afecten a todo el conjunto. $\mathrm{Y}$ en contrapunto, encontramos imposible agregar dichos elementos en un modelo de "fundamentación racional", deductiva, pensado para resoluciones universales y objetivas. La ética del cuidado, aun siendo un modelo menos "práctico", recupera dichas dimensiones, alertando a la vez de la imperfección de los modelos tradicionales $(7,18)$.

\section{De la teoría a la práctica en la profesión enfer- mera. Limitaciones de la ética del cuidado}

Se debe reconocer que hasta ahora la ética del cuidado tiene sus propias limitaciones. Una de ellas, y por la que ha sido objeto de múltiples críticas, es la dificultad para llevarla a la práctica(21). A menudo, los modelos y teorías de enfermería no pueden llevarse a cabo de una manera real y aplicada, bien por su complejidad, por su naturaleza teórica o simplemente porque no se ha profundizado en su contenido. Pero son propuestas indispensables por su fundamento teórico, que marca y determina el ideal del quehacer profesional y que permite el avance de la ciencia en sí(22).

En efecto, en la actualidad varias corrientes creen que la ética del cuidado no puede convertirse en la única ética de enfermería por su naturaleza teórica, inmensurable y su falta de desarrollo, y por lo tanto se requiere el conocimiento de los demás modelos bioéticos(8). Por esto, tal vez su importancia radique simplemente en darla a conocer, en divulgarla, porque no cabe duda que es el corazón, el alma de nuestra práctica profesional.

Si profundizamos en la verdadera dificultad que tiene este modelo para ser llevado al ámbito asistencial, nos encontraríamos con su potente implicación humanística que, en contraposición, permite cuidar a las personas junto a sus problemas, en vez de centrarnos únicamente en el problema a tratar(23). Pero, por otro lado, esto mismo dificulta su propia expresión práctica y su falta de indicadores para la evaluación. 
Entonces la ética del cuidado, no es tanto una manera o un protocolo de cómo ejercer el cuidado, sino un modo o una actitud para llevarlo a cabo. Dicho de otro modo, la práctica de la ética del cuidado se concreta por medio de la relación terapéutica(8), aunque también se exija una lógica competencia técnica $(5,24)$.

¿Puede entonces enseñarse a cuidar dentro de la ética del cuidado? La respuesta es sí, pero se deben reconocer sus complicaciones. El aprendizaje del concepto sería fácil, pero el aprendizaje para proporcionarlo de manera honesta no está exento de dificultad(10). No se trata solamente de enseñar explícitamente el modelo, sino de transmitir una actitud global. A lo que se transmite de forma tácita se le llama "oculto" y no siempre coincide en todos sus sentidos con lo evidente. Es de esta manera como se pueden enseñar los valores profesionales, además de enseñarlos de manera formal(23).

Básicamente, los fundamentos de la ética del cuidado pueden describirse a partir de cinco ejes:

- El concepto del ser como ser relacional: en nuestra vida necesitamos de los demás y de las relaciones con ellos que nos permitan darnos a ellos, tanto para sobrevivir como para autorrealizarnos.

- Un enfoque sensitivo al contexto: lo que incorpora una observación del otro y su entorno, para identificar la totalidad de sus necesidades.

- La diferenciación entre cuidado y preocupación: esto implica que exista atención tanto hacia las personas con las que tenemos relaciones personales, como hacia aquellas con las que no compartimos dichos lazos de unión.

- La prioridad del sentir sobre el razonar: este es el punto clave de la ética del cuidado y que le diferencia de otros modelos: dar relevancia justa a los sentimientos y afectos.

- La opción por los dilemas reales sobre los dilemas hipotéticos: es decir, personalizar y singularizar las resoluciones sin equipararlos con casos hipotéticos o anteriores(16).
En general, estamos acostumbrados a juzgar las acciones tachándolas de malas o buenas, correctas o incorrectas, sobre todo cuando se trata de casos hipotéticos, sin admitir excepciones, por lo que, en definición, nuestras resoluciones u opiniones no debieran cambiar según la situación. En la práctica, su consideración puede cambiar desde este nuevo punto de vista. No se trata de relativismo moral, sino de la parte de un reconstruido entendimiento moral que permite, renunciando al mero juicio, advertir la determinación psicológica y moral del comportamiento humano. En definitiva, se trata de tener en cuenta la preocupación moral por el sufrimiento y el dolor(17). No de cambiar las decisiones éticas según utilidad, sino que de admitir que el contexto de cada situación hace necesario individualizar las respuestas a los supuestos éticos. No se trata pues de subjetivismo, sino de una actitud hacia el otro. Por esto, la ética del cuidado no está compuesta por principios universales e inamovibles y más que un método se trata de una actitud para la deliberación.

\section{Actitudes y elementos necesarios}

Recapitulando: es necesario para el buen desempeño de nuestras funciones un concepto de cuidado que incluya tanto su significado técnico como transcendental, y que incluya tanto la participación profesional como la del paciente. Por otro lado, la ética del cuidado aportaría los valores o principios esenciales en los que se debería basar nuestro quehacer profesional. Pero, ¿cuáles son entonces las actitudes necesarias para ello? Las virtudes necesarias que podrían exigirse, acorde con las concepciones del cuidado y la ética del cuidado, serían la empatía, la prudencia, la compasión, el afecto, la coherencia y la escucha.

Según Carol Taylor, son tan solo seis las características o aptitudes básicas para llegar a ser un buen "cuidador": "el afecto (experiencia de un sentimiento positivo hacia el paciente), la cognición (capacidad intelectual y técnica de identificar lo esencial para el bienestar del paciente), la volición (compromiso interno para movilizar los recursos personales y técnicos que aseguren el bienestar del paciente), la imaginación (emplear creativamente los recursos personales para relacionarse empáticamente con el paciente y entender su situación existencial particular, así como sus 
necesidades vitales), la motivación (flujo de energía aplicado al acto de cuidar, refleja el altruismo en el ejercicio de la profesión y el deseo de actuar en consecuencia), y finalmente la expresión (demostrar genuinamente la intención de ayuda mediante el uso de la estrategia de comunicación verbal y no verbal)"(5).

Finalmente, tras un periodo de aprendizaje, para llevar todo esto a la práctica, debemos concienciarnos de que somos nuestro propio y mejor instrumento, ampliando nuestros recursos internos y eliminando la creencia de que nuestra única función es administrar medicación, revindicando que existen también los llamados "cuidados invisibles" (25).

Por otro lado, debemos ser conscientes de que para prestar cuidados excelentes se necesita tiempo. Y debemos insistir en ello como colectivo. Cuando no hay tiempo, nos vemos obligadas, lógicamente, a priorizar en las tareas técnicas, que en general suelen ser tareas interdependientes o delegadas y no propias, y esto nos impide realizar nuestra verdadera labor(25).

Por último, debemos procurar un ambiente cómodo y estable, para desarrollar el compromiso profesional. Las enfermeras debemos trabajar en un entorno que facilite la relevancia de nuestra propia aportación, que incluiría recursos materiales y humanos(25).

\section{Conclusiones}

Es indudable que la enfermería es una profesión que posee una carga ética importante. No obstante, por el momento, está carente de una visión bioética definida, la cual ayudaría a completar su propia definición profesional y además serviría a los profesionales como instrumento decisorio. La ética del cuidado está íntimamente relacionada con la enfermería y, aunque no es exclusiva de ella, podría funcionar como pilar fundamental para determinar su propio modelo.

En lo que al cuidado se refiere, podemos diferenciar dos aspectos básicos: el técnico, cuyos objetivos están bien definidos y regulados por medio de tareas, y el aspecto del cuidado como bien interno, el que proporciona sentido a la profesión, así como plena satisfacción de los usuarios. Es un aspecto más difícil de definir, de llevar a cabo y de cuantificar, pero no por ello menos importante. Por esto es necesario ampliar la visión del concepto, dejando de lado su dualidad; cuidando a la persona como se merece, de manera holística, teniendo en cuenta todas sus dimensiones que van más allá de su mera corporalidad. Como profesionales tenemos el compromiso moral de ejercer el cuidado en todos sus aspectos y debemos exigir a los gestores que nos dejen llevar a cabo nuestra misión.

Tal vez, la ética del cuidado no tenga una aplicación práctica fácil, por sus valores humanistas y su dificultad en la medición. Pero, ¿por qué hay que medirlo todo? ¿Carece de utilidad la ética del cuidado? Simplemente su importancia radica en que constituye una apertura hacia otro modo de ver la vida y la influencia de nuestras decisiones.

Son muchas y distintas las actitudes que debemos trabajar para conseguir aplicarlas en las relaciones, pero nuestro mejor instrumento somos nosotros mismos, aunque lógicamente también es muy necesario exigir una competencia técnica, tiempo y recursos materiales y humanos.

Valdría la pena tomarse un respiro para valorar el propio desarrollo de la profesión, replantear lo que nos falta, lo que debemos profundizar, y, con ello, recuperar su verdadero fundamento: el cuidado. La ética del cuidado puede ayudarnos a recuperar la identidad propia de nuestra profesión.

\section{Agradecimientos}

A Elena Postigo, María Teresa Delgado y Rogelio Altisent, por el esfuerzo hacia su profesión, generosidad, ayuda, apoyo y colaboración. 


\section{Referencias}

1. Santacruz Caicedo MC. Ética del cuidado. Revista Facultad Ciencias de la Salud, Universidad de Cauca 2006; 8(2): 45-51.

2. Prieto Parra GI. Perspectiva bioética del cuidado de enfermería. Revista Enfermería Universitaria 2007; 4(3): 21-26.

3. Llanos Peña F. Bioética y ética del cuidado. Hygia 1999; 41(3).

4. Granero Molina J, Jiménez López FR, Fernández Sola C. Implicaciones éticas en la aplicación de los procedimientos especiales enfermeros. Metas Enferm 2005; 8(5): 56-63.

5. Montoya GJ. La ética del cuidado en el contexto de la salud sexual y reproductiva. Acta Bioethica 2007; 13(2): 168-175.

6. Barrio Cantalejo IM. Carol Gilligan y la ética del cuidado. Un referente para la ética de enfermería. Enferm Clin 1999; 9(2): 71-76.

7. Feito Grande L. Los cuidados en la ética del siglo XXI. Enferm Clin 2005; 15(3): 167-174.

8. Davis, AJ. Las dimensiones éticas del cuidar en enfermería. Enferm Clin 1999; 9(1): 21-28.

9. Real Academia Española. Diccionario de la lengua española. 22a ed. Madrid: Espasa-Calpe; 2001.

10. Davis AJ. El cuidar y la ética del cuidar en el siglo XXI: qué sabemos y qué debemos cuestionar. Collegi Oficial d'Infermeria de Barcelona 2006 (Acceso 11 de diciembre de 2011).

11. Alberdi Castell RM, Cuxart Ainaud N. Cuidados, enfermeras y desarrollo profesional: Una reflexión sobre las bases del ejercicio profesional. Presencia 2005; 1(2).

12. Calvo-Gil MJ, Ayala-Valenzuela R, Holmqvist-Curimil M, Molina-Díaz C. Aspectos axiológico-culturales de la ética y el cuidado. Acta Bioethica 2011; 17(1): 133-141.

13. Gándara M. Ética, poder y deber: de la ética del cuidado al poder del deber de cuidar. Rev rol enferm 2008; 31(1): 18-24.

14. De la Torre Díaz J. Mujer, mujeres y bioética. Madrid: Universidad Pontificia Comillas; 2010.

15. Alvarado García A. La ética del cuidado. Aquichan 2004; 4(4): 30-39.

16. Bonilla Ballesteros AR. Análisis comparativo de cinco teorías sobre el desarrollo moral. Tesis doctoral. Bogotá: Pontificia Universidad Javeriana; 2005.

17. Gilligan C. In a different voice: psychological theory and women's development. Harvard University Press; 1983.

18. Feito Grande L. Enfoques consecuencialistas: La ética del cuidado. Material docente, Master en Bioética, Universidad de Las Palmas; 2006.

19. Feito Grande L. La Ética del Cuidado como modelo de la ética enfermera. Metas Enferm 2005; 8(8): 14-18.

20. Hernán Baeza R. Valores y comunicación. Ética del cuidado. Medicina General 2001; 31: 113-120.

21. Cibanal JL, Siles González J. El mundo de los valores en el cuidado enfermero. Enfermería Cientifica 1998; $194-195$.

22. López M, Santos S, Varez S, et al. Reflexiones acerca del uso y utilidad de los modelos y teorías de enfermería en la práctica asistencial. Enferm Clin 2006; 16(4): 214-217.

23. Escuredo Rodríguez B. Humanismo y tecnología en los cuidados de enfermería desde la perspectiva docente. Enferm Clin 2003; 13(3): 164-170.

24. Arroyo Gordo MP. Dimensiones éticas en los cuidados enfermeros metas. Metas Enferm 2007; 10(6): 3.

25. Alberdi Castell RM, Arriaga Piñeiro E, Zabala Blanco J. La ética del cuidado: una propuesta con futuro para las enfermeras y las empresas sanitarias. Rev rol enferm 2006; 29(3): 31-38.

Recibido: 6 de mayo de 2014

Aceptado: 8 de mayo de 2014 